\title{
Laser Processing of Silicon Suboxide for the Fabrication of Multilevel Fused Silica Diffractive Phase Elements
}

\author{
Lukas Janos Richter, Clemens M. Beckmann, Jörg Meinertz, Jürgen Ihlemann \\ Laser-Laboratorium Göttingen e.V., Hans-Adolf-Krebs-Weg 1, 37077 Göttingen, Germany \\ E-mail: juergen.ihlemann@llg-ev.de
}

\begin{abstract}
UV-absorbing silicon suboxide $\left(\mathrm{SiO}_{\mathrm{x}}, \mathrm{x} \approx 1\right)$ films can be patterned by excimer laser ablation and subsequently oxidized to fused silica by thermal annealing. This two-step process allows for the fabrication of microstructured components made entirely of fused silica. For example, diffractive optical elements with two phase-quantized levels (binary DOEs) can be produced via structured rear-side ablation of a thin film of silicon suboxide on a fused silica substrate followed by oxidation. Such phase elements can be used as projection masks for precise laser microfabrication, e.g. for parallel nano hole drilling. In addition to these binary patterns, multilevel phase structures are produced by repeating the ablation step after recoating the structured surface with additional layers of silicon suboxide. A diffractive phase element in form of a three-level line grating exhibits a diffraction efficiency of more than $60 \%$ in the +1 st order. This efficiency can be enhanced by increasing the number of recoating and ablation steps and, thus, the number of phase levels. Within the levels, a surface roughness of $R_{a} \approx 3 \mathrm{~nm}$ is obtained.
\end{abstract}

DOI: $10.2961 /$ jlmn.2018.03.0018

Keywords: Silicon suboxide, excimer laser, fused silica, thin film patterning, diffractive elements, multilevel phase elements

\section{Introduction}

Diffractive phase elements (DPE) can realize optical functionalities that are difficult or impossible to achieve with conventional optics. Thus, they offer a high potential for applications in illumination, imaging, microscopy and laser processing $[1,2]$. Compared to diffractive amplitude elements like delicate and easily degrading chrome on quartz gratings, they are characterized by higher diffraction efficiency, higher transmission, and enhanced durability. They can be implemented in form of a height profile corresponding to the required phase modulation patterned onto an optically transparent material. For the fabrication of polymeric DPE, several replication techniques like embossing, molding, and casting are available [3]. Excimer laser ablation has been applied for the fabrication of pixelated DPE in polymer material [4]. Multilevel elements in polymers can be obtained using halftone masks [5]. Femtosecond laser ablation is applied for other transparent materials like indium tin oxide (ITO) films [6]. For many heavy-duty applications, fused silica is the optimum material due to its high transparency from the deep UV to the near IR and its chemical and thermal stability. Conventionally, elements from fused silica are made by a complex and expensive lithographic process including reactive ion etching [7]. The precise laser machining of fused silica is rather difficult. Fabrication of DPE by laser ablation has been investigated using $\mathrm{CO}_{2}$ lasers [8], excimer lasers [9], and $F_{2}$-lasers [10]. Drawbacks are limited spatial resolution using a laser wavelength of $10.6 \mu \mathrm{m}$, lack of precise depth control at $248 \mathrm{~nm}$ or $193 \mathrm{~nm}$, and complexity of the optical system at $157 \mathrm{~nm}$, respectively. Furthermore, laser induced backside wet etching (LIBWE) has been tested for the fabrication of fused silica phase elements [11]. We have developed a method that requires just a few laser pulses and combines high lateral resolution and precise depth control: a UVabsorbing silicon suboxide film $\left(\mathrm{SiO}_{\mathrm{x}}, \mathrm{x} \approx 1\right)$ is deposited on a fused silica substrate, patterned by structured ablation, and subsequently oxidized to UV-transparent $\mathrm{SiO}_{2}[12,13]$. This way, binary (two-level) phase elements have been fabricated for applications in fs-laser micromachining [14] or for the laser based fabrication of templates for the ordered arrangement of nanoparticles [15]. To obtain high precision of the created surface profile, the $\mathrm{SiO}_{\mathrm{x}}$-film is patterned by rear side ablation, i.e. the laser pulse is guided from the backside through the substrate to the film. This means that the film is either completely ablated or not at all, leading automatically to a two-level profile. To generate a 3- or 4- phase level element, either multi-pulse front side ablation has to be used leading to a reduction in precision [16], or the phase element has to be composed of two binary elements [17]. In this paper we describe instead the fabrication of monolithic multilevel phase elements by repeating the ablation step after recoating the surface with additional layers of silicon suboxide leading to better depth and edge control and reduced roughness compared to [16].

\section{Fabrication of phase elements}

Binary phase elements are fabricated according to the following scheme:

1. A UV-absorbing coating of silicon suboxide $\left(\mathrm{SiO}_{\mathrm{x}}\right)$ is deposited on a fused silica substrate by e-beam evaporation of silicon monoxide (Laseroptik, Garbsen, Germany). The deposition rate is about $1 \mathrm{~nm} / \mathrm{s}$. 
2. The $\mathrm{SiO}_{\mathrm{x}}$-coating is patterned by ArF-excimer laser ablation to form the desired phase structure. The laser wavelength is $193 \mathrm{~nm}$, the pulse duration $20 \mathrm{~ns}$.

3. The SiOx-material is oxidized to UV-transparent silicon dioxide $\left(\mathrm{SiO}_{2}\right)$ by thermal annealing for several hours (depending on thickness) at $1000^{\circ} \mathrm{C}$ in air.

Fig. 1 displays the transmission spectra of a $220 \mathrm{~nm}$ thick film before $\left(\mathrm{SiO}_{\mathrm{x}}\right)$ and after $\left(\mathrm{SiO}_{2}\right)$ thermal oxidation. During oxidation the film thickness increases by about $30 \%$ due to oxygen incorporation while the material is maintaining its density [18]. This has to be taken into account in the design parameters. For a two-level phase element with suppressed zero diffraction order, a profile depth (i.e. thickness of the layer) of $d=\lambda /[2(n-1)]$ ( $\lambda$ operation wavelength, $\mathrm{n}$ refractive index) and a duty cycle of 0.5 (equal area at both levels) are required. Ablation is performed with an ArF excimer laser (Coherent LPXpro 220, beam size $8 \mathrm{~mm} \times 24 \mathrm{~mm}$, max. repetition rate $200 \mathrm{~Hz}$ ) by mask projection in a rear side configuration irradiating the $\mathrm{SiO}_{\mathrm{x}}$-film through the transparent fused silica substrate. For the generation of line patterns or crossed line patterns, the laser illuminated slit mask is imaged by a fused silica cylindrical lens of $63 \mathrm{~mm}$ focal length with 10:1 demagnification. The slit mask is made from two steel blades with adjustable distance. A single ArF laser pulse with a fluence of $\sim 500 \mathrm{~mJ} / \mathrm{cm}^{2}$ is sufficient to ablate a line with the desired width of $\sim 5-100 \mu \mathrm{m}$ and a length of $20 \mathrm{~mm}$. Remaining spaces between ablated lines can as well be as narrow as $5 \mu \mathrm{m}$ or even less. Height profiles are recorded with a confocal microscope (Sensofar) and an atomic force microscope (Park Systems XE-150). One- and two-dimensional binary patterns are shown in fig. 2 .

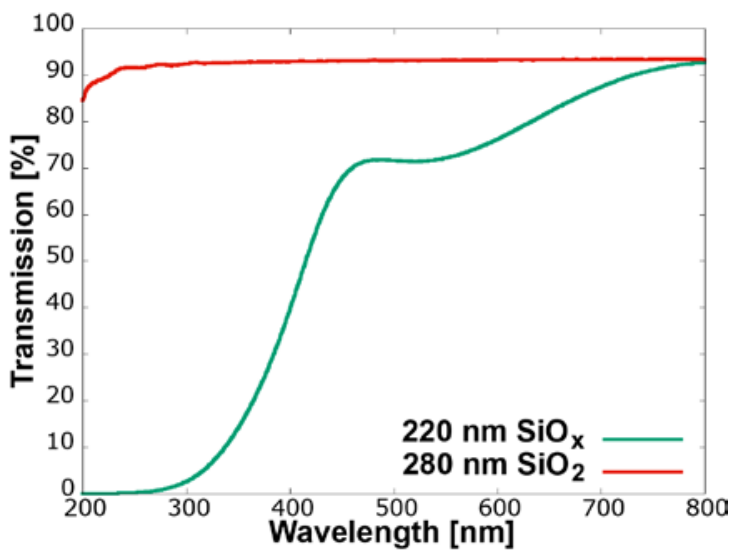

Fig. 1 Transmission spectra of a $220 \mathrm{~nm}$ thick $\mathrm{SiO}_{\mathrm{x}}$ film on fused silica before and after oxidation to $\mathrm{SiO}_{2}$. An increase of thickness of $27 \%$ to $280 \mathrm{~nm}$ is observed during oxidation.

For the fabrication of multilevel elements the steps of film deposition and film ablation are alternately repeated. This is shown in fig. 3 for the simplest case of a three-level element. After patterning of the first film, a second coating (of the same or different thickness) is deposited over the structured film. With the second ablation process, depending on position, the second film or both films are locally removed. For exact positioning of the second exposure with respect to the previous one, a camera based viewing system is used. During the final annealing step the whole film material is oxidized to $\mathrm{SiO}_{2}$. This way a stair pattern representing a blaze profile is generated (fig. 4). The slight variation of the step widths is due to the limitations in position repeatability between the first and the second exposure.

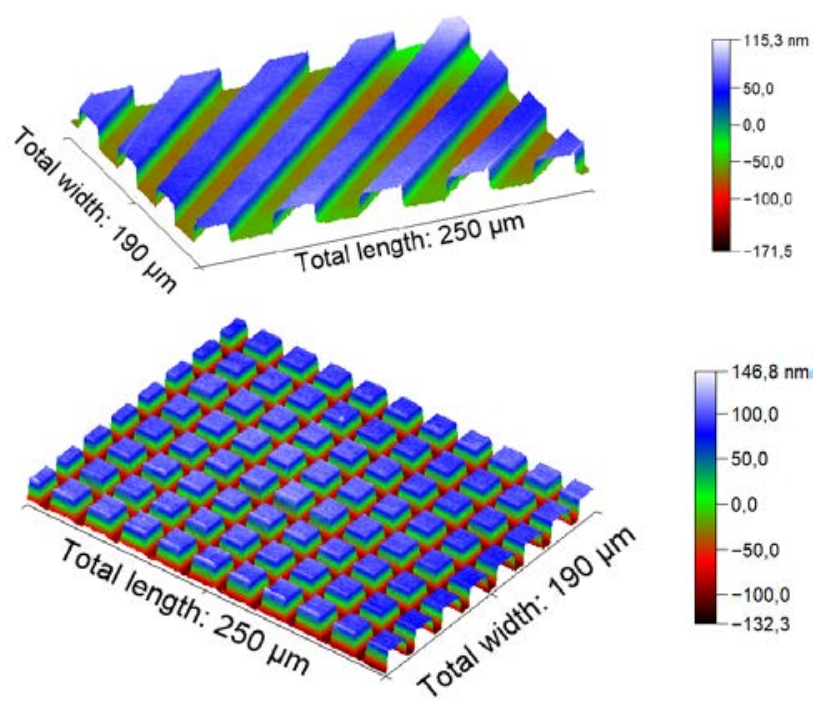

Fig. 2 Height profiles of two-level phase elements recorded with a confocal microscope. Top: linear phase grating $(40 \mu \mathrm{m}$ period); Bottom: crossed phase grating ( $25 \mu \mathrm{m}$ period).
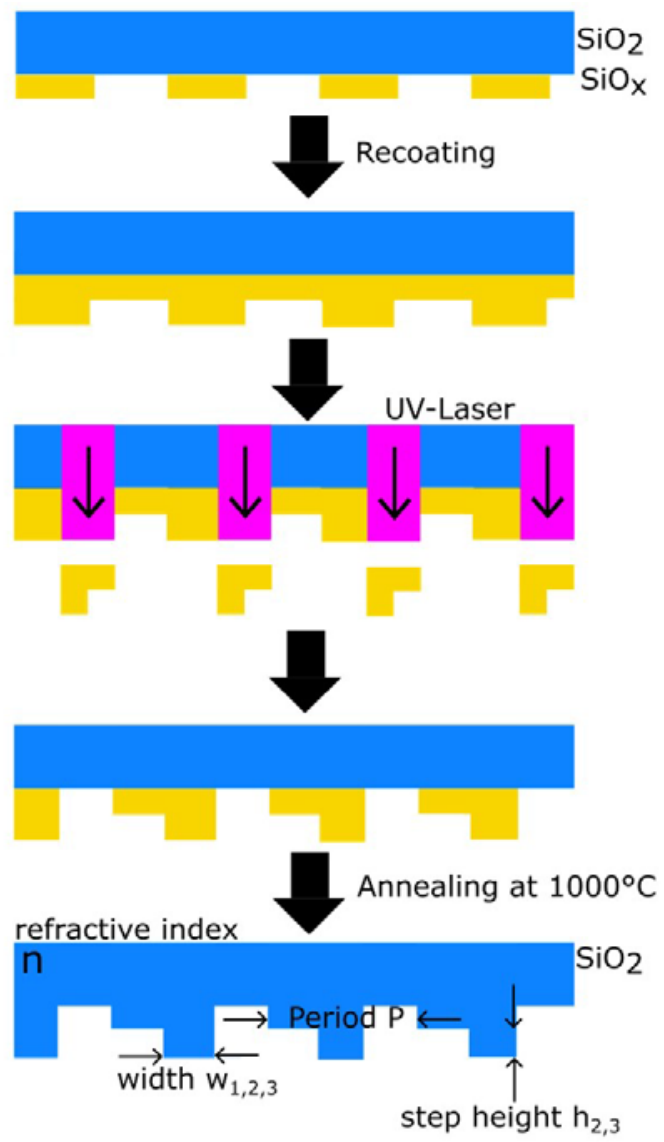

Fig. 3 Process steps of the fabrication of a three-level phase mask with blaze-like structure 


\section{Diffraction efficiency}

To characterize the optical functionality of the threelevel blaze like structure shown in fig. 4, the diffraction efficiency has been measured at three wavelengths $(633 \mathrm{~nm}$, $405 \mathrm{~nm}$, and $308 \mathrm{~nm}$ ). The efficiency D.E. $=I_{m} / I_{\text {tot }}$ is determined by measuring the beam intensity $I_{m}$ in diffraction order $m$ and the total intensity $I_{\text {tot }}$ directly behind the sample, before the orders separate. At $308 \mathrm{~nm}$, pulse energies are measured with a pyroelectric detector (Ophir PE10BF), at $405 \mathrm{~nm}$ and $633 \mathrm{~nm}$, the beam power is measured by photodiodes (Ophir PD-300).

The measured data are compared to the theoretically expected diffraction efficiencies, calculated by using the data for step heights $h_{i}$ and step widths $w_{i}$ taken from AFM measurements (fig. 4). With these data, the complex transmission function $t(x)$ within one period $P$ of the three-level phase-quantized grating reads

$$
t(x)=\sum_{i=1}^{3} e^{i \frac{2 \pi}{\lambda}(n-1) h_{i}} \operatorname{rect}\left(\frac{x-x_{i}}{w_{i}}\right)
$$

where $\lambda$ is the wavelength, $n$ the refractive index, and $x_{i}$ denotes the center of the i-th step. The variation of the refractive index $n(\lambda)$ over the investigated spectral range is taken into account by using tabulated data for fused silica [19]. The diffraction efficiencies are obtained by writing $t(x)$ as a Fourier series:

$$
t(x)=\sum_{m=-\infty}^{\infty} t_{m} e^{2 \pi i m \frac{x}{P}}
$$

Then, the diffraction efficiency of the grating for the $\mathrm{m}$ th transmitted diffraction order is given by the absolute value squared of the m-th Fourier coefficient. Thus, one obtains [20]:

$$
\text { D.E. }=\left|t_{m}\right|^{2}=\left|\frac{1}{P} \int_{-P / 2}^{P / 2} t(x) e^{-2 \pi i m \frac{x}{P}} d x\right|^{2}
$$
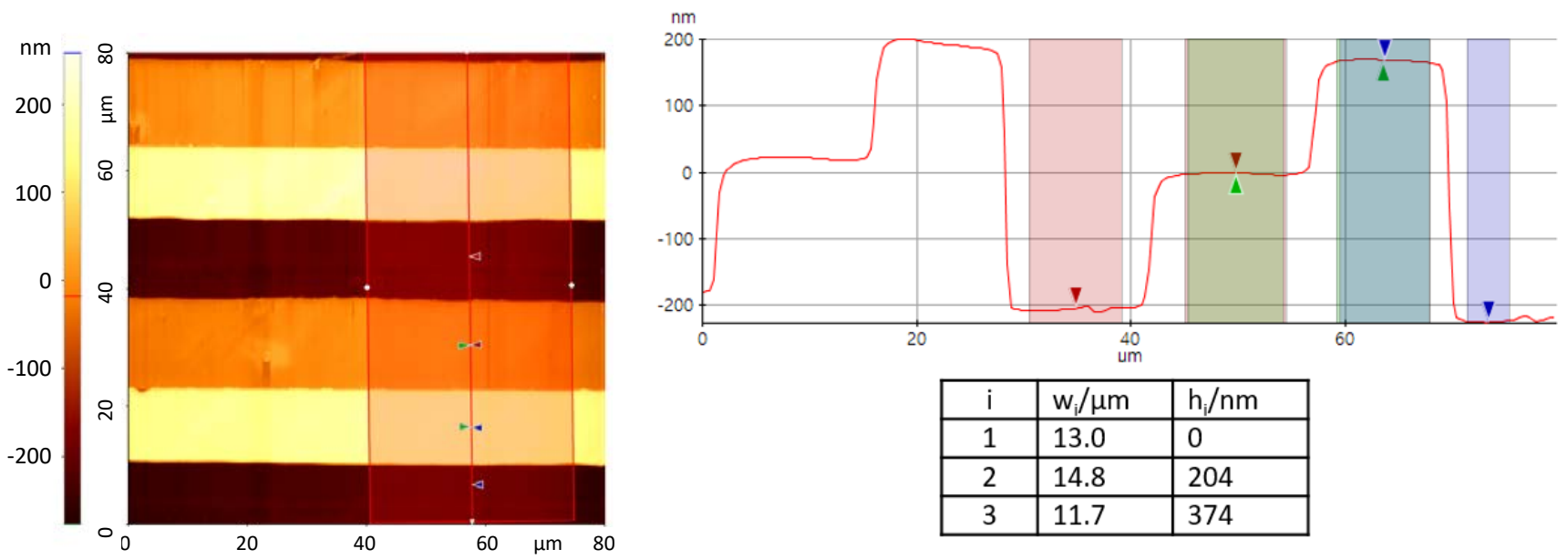

Fig. 4 Three-level phase element fabricated according to the scheme of fig. 3. Laser parameters for both ablation steps: $193 \mathrm{~nm}$, $450 \mathrm{~mJ} / \mathrm{cm}^{2}, 1$ pulse. Image taken by atomic force microscopy.

Left: False color representation of the height profile. Right: Cross section through the area indicated on the left side.

The comparison of measured and simulated diffraction efficiencies in fig. 5 shows excellent agreement. The data demonstrate that the Blaze effect is effective already in this three-level configuration: wheras around $300 \mathrm{~nm} 2 / 3$ of the incoming light is diffracted into the +1 . order, the 0 . order is suppressed to less than $3 \%$.

A significant advantage of this method based on film ablation compared to fused silica bulk ablation is the obtained low roughness. As the film-substrate interface behaves as predetermined breaking point, the smoothness of the substrate or the coated substrate is always restored. Thus, roughness values of $R_{a} \approx 3 \mathrm{~nm}$ are obtained (fig. 6), so that scattering losses of these optical elements due to surface roughness are intrinsically minimized. In contrast, using (front side) ablation of bulk fused silica at $157 \mathrm{~nm}$, a RMS surface roughness of $13 \mathrm{~nm}$ is obtained [10]. 


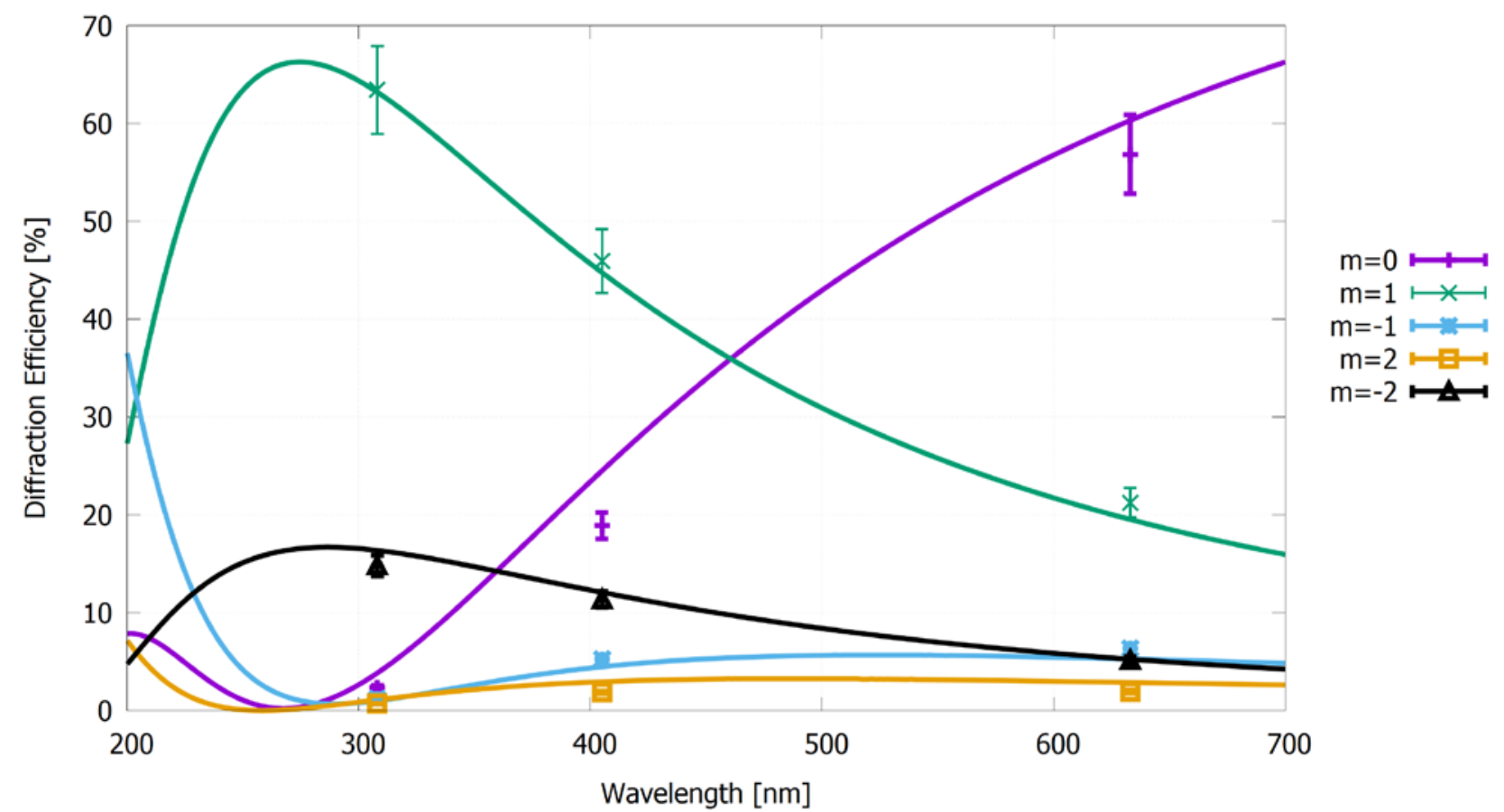

Fig. 5 Measured (data points) and simulated (lines) diffraction efficiencies for the three-level structure shown in fig. 4. The diffraction efficiency is shown for the orders $m=-2$ to $m=2$

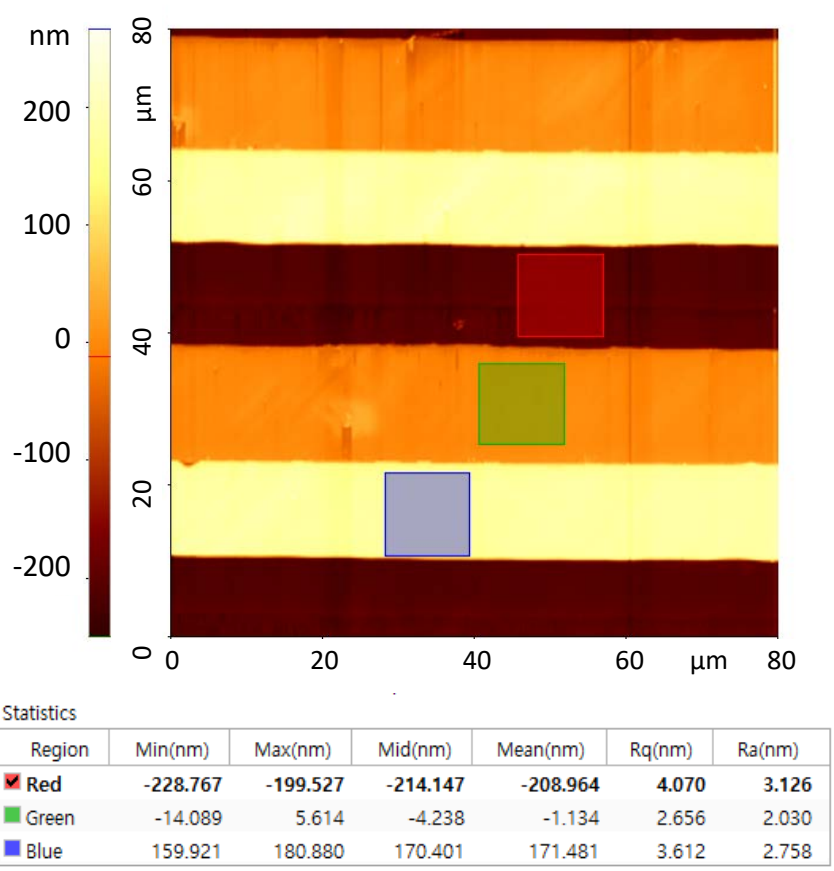

Fig. 6 Roughness measured in $10 \mu \mathrm{m} \times 10 \mu \mathrm{m}$ large areas on the three levels of a diffractive phase element

\section{Conclusion}

UV-absorbing $\mathrm{SiO}_{\mathrm{x}}$-films can be patterned by excimer laser ablation und subsequently oxidized to $\mathrm{SiO}_{2}$. Phase elements with precisely controlled phase shift are obtained this way. Multilevel phase elements are fabricated by recoating and processing of prestructured surfaces. Even a three-level element can be designed for high diffraction efficiency in specific diffraction orders. The fabrication of four-level blaze-structures can even increase the diffraction efficiency. This patterning process is simple and fast (only one laser pulse per line and depth level is required) and cost-effective (a low power tabletop excimer laser with suitable beam delivery is sufficient). The annealing process takes several hours, but can be applied simultaneously to many glass plates, reducing significantly the fabrication time of DPE.

\section{Acknowledgments}

Financial support by the EU and the state of Lower Saxony through EFRE is gratefully acknowledged.

\section{References}

[1] J. Turunen, F. Wyrowski eds.: "Diffractive Optics for Industrial and Commercial Applications”, (Akademie Verlag, Berlin, 1997).

[2] D.C. O’Shea, T.J. Suleski, A.D. Kathman, D.W. Prather: "Diffractive Optics: Design, Fabrication and Test”, SPIE tutorial texts, v. TT62 (2004).

[3] M.T. Gale: Microelectronic Engineering, 34, (1997) 321.

[4] G.P. Behrmann, M.T. Duignan: Appl. Opt., 36, (1997) 4666.

[5] F. Quentel, J. Fieret, A.S. Holmes, S. Paineau: Proc. SPIE 4274, (2001) 420.

[6] M. Baum, J. Strauß, F. Grüßel, I. Alexeev, M. Schmidt: J. Opt., 16, (2014) 125706. 
[7] E.B. Kley: Microelectronic Engineering, 34, (1997) 261.

[8] K.L. Wlodarczyk, N.J. Weston, M. Ardron, D.P. Hand: Opt. Expr., 24, (2016) 1447.

[9] J. Bekesi, D. Schäfer, J. Ihlemann, P. Simon: Proc. SPIE 4977A, (2003) 235.

[10] M.L. Ng, P.R. Herman, A.H. Nejadmalayeri, and J. Li: J. Phys. Conf. Ser., 59, (2007) 696.

[11] G. Kopitkovas, T. Lippert, C. David, A. Wokaun, J. Gobrecht: Microelectronic Engineering, 67, (2003) 438.

[12] M. Schulz-Ruhtenberg, J. Ihlemann, J. Heber: Appl. Surf. Sci., 248, (2005) 190.

[13] T. Fricke-Begemann, J. Meinertz, R. WeichenhainSchriever, J. Ihlemann: Appl. Phys. A, 117, (2014) 13.
[14] J. Ihlemann, J.-H. Klein-Wiele, J. Békési and P. Simon: Journal of Physics: Conference Series 59, 449 (2007).

[15] R. Karstens, A. Gödecke, A. Prießner, J. Ihlemann: Optics and Laser Technology 83, 16 (2016)

[16] J. Ihlemann, J. Meinertz, G. Danev: Appl. Phys. Lett., 101, (2012) 091901.

[17] J. Kaakkunen, J. Bekesi, J. Ihlemann, P. Simon: Appl. Phys. A, 101, (2010) 225.

[18] G. Hass, C.D. Salzberg: J. of the Optical Society of America, 44, (1954) 181.

[19] I.H. Malitson: J. of the Optical Society of America, 55, (1965) 10.

[20] J. Jahns, S. Helfert "Introduction to Micro- and Nanooptics”, (Wiley-VCH, Weinheim, 2012) p.137.

(Received: June 21, 2018, Accepted: November 11, 2018) 